\title{
Enterocolite Neutropênica em Paciente com Câncer de Mama em Uso de Quimioterapia Adjuvante: Relato de Caso
}

https://doi.org/10.32635/2176-9745.RBC.2021v67n1.1188

\author{
Neutropenic Enterocolitis in a Breast Cancer Patient using Adjuvant Chemotherapy: Case Report \\ Enterocolitis Neutropénica en Paciente con Cáncer de Mama que Usa Quimioterapia Adyuvante: Reporte de Caso
}

\author{
Luana Santana Bacelar'; Martamaria de Souza Ferraz Ribeiro²; Caroline Chagas Lopes ${ }^{3}$; Elisama Andrade Miranda4; Rosa Malena \\ Fagundes Xavier ${ }^{5}$; Maria Teresita Bendicho ${ }^{6}$
}

\section{RESUMO}

Introduçáo: A enterocolite neutropênica (EN) consiste em ulceração ou necrose da mucosa do ceco, íleo terminal e cólon ascendente, sendo uma condição clínica ocasionada como evento adverso de medicamentos, principalmente em esquemas quimioterápicos. Por ser uma condição com alto índice de mortalidade, o presente relato tem como objetivo contribuir significativamente para discussões que envolvem a EN e a participação da equipe multiprofissional no desfecho clínico. Relato do caso: Paciente do sexo masculino, 75 anos, com diagnóstico de câncer de mama, evoluindo com EN após tratamento com quimioterapia adjuvante. A presença de comorbidades e a idade foram os principais fatores complicadores do quadro de tiflite. Por ser uma toxicidade importante e que pode levar à piora do quadro clínico do paciente com câncer, abordar esse tema é fundamental para um diagnóstico mais rápido, com possibilidade de medidas preventivas. Conclusáo: Sendo assim, em virtude do notório aumento dos casos de EN, aponta-se como perspectiva a qualificação da equipe de saúde para a inserçáo de profissionais ainda mais especializados, capazes de contribuir e identificar os sinais e sintomas relacionados com toxicidades hematológicas, resultado de tratamentos quimioterápicos.

Palavras-chave: Enterocolite Neutropênica/tratamento farmacológico; Efeitos Colaterais e Reaçóes Adversas Relacionados a Medicamentos; Neoplasias da Mama Masculina; Quimioterapia Adjuvante; Equipe de Assistência ao Paciente.

\section{ABSTRACT}

Introduction: Neutropenic enterocolitis (NE) consists of ulceration or necrosis of the mucosa of the cecum, terminal ileum, and ascending colon, being a clinical condition caused by an adverse drug event, mainly in chemotherapy regimens. As it is a high mortality rate condition, this report aims to contribute significantly to discussions involving NE and the participation of the multidisciplinary team in the clinical outcome. Case report: This is a 75-year-old male patient diagnosed with Breast Cancer, who developed EN after treatment with adjuvant chemotherapy. The presence of comorbidities and age were the main complicating factors in typhlitis. As it is an important toxicity and can lead to a worsening of the clinical condition of cancer patients, addressing this issue is essential for a faster diagnosis with the possibility of preventive measures. Conclusion: Therefore, in view of the notorious increase of cases of NE, the perspective of the qualification of the health team is pointed out, for the inclusion of even more specialized professionals capable of contributing and identifying the signs and symptoms related to hematological toxicities, result of chemotherapy treatments. Key words: Enterocolitis, Neutropenic/drug therapy; Drug-Related Side Effects and Adverse Reactions; Breast Neoplasms, Male; Chemotherapy, Adjuvant; Patient Care Team.

\section{RESUMEN}

Introducción: La enterocolitis neutropénica (EN) consiste en la ulceración o necrosis de la mucosa del ciego, íleon terminal y colon ascendente, siendo una condición clínica causada por un evento adverso farmacológico, principalmente en regímenes de quimioterapia. Al tratarse de una afección con una alta tasa de mortalidad, este informe tiene como objetivo contribuir de manera significativa a las discusiones que involucran al EN y la participación del equipo multidisciplinario en el resultado clínico. Relato del caso: Paciente masculino, 75 años, diagnosticado de cáncer de mama, que desarrolló EN después del tratamiento con quimioterapia adyuvante. La presencia de comorbilidades y la edad fueron los principales factores de complicación en Tiflite. Como se trata de una toxicidad importante y puede conducir a un empeoramiento de la condición clínica de los pacientes con cáncer, abordar esta cuestión es fundamental para un diagnóstico más rápido con la posibilidad de medidas preventivas. Conclusión: Por tanto, ante el notable incremento de casos de EN, se apunta la perspectiva de la calificación del equipo de salud, para la inclusión de profesionales aún más especializados capaces de aportar e identificar los signos y síntomas relacionados con las toxicidades hematológicas, un resultado de los tratamientos de quimioterapia.

Palabras clave: Enterocolitis Neutropénica/tratamiento farmacológico; Efectos Colaterales y Reacciones Adversas Relacionados con Medicamentos; Neoplasias de la Mama Masculina; Quimioterapia Adyuvante; Grupo de Atención al Paciente.

\footnotetext{
1,5,6Universidade do Estado da Bahia (UNEB). Departamento de Ciências da Vida. Salvador (BA), Brasil. E-mails: luanabacelar.farma@gmail.com; rxavier@uneb.br; mtbendicho@gmail.com. Orcid iD: https://orcid.org/0000-0003-4373-826X; Orcid iD: https://orcid.org/0000-0002-3203-8949; Orcid iD: https://orcid.org/00000001-8234-1199

2,3,4 Hospital Santa Izabel. Salvador (BA), Brasil.E-mails: martasfribeiro@gmail.com; kau_chagas@hotmail.com; elisamaandrade.m@gmail.com. Orcid iD: https://orcid. org/0000-0001-8300-6229; Orcid iD: https://orcid.org/0000-0002-3190-9597; Orcid iD: https://orcid.org/0000-0003-0458-7817

Endereço para correspondência: Luana Santana Bacelar. Departamento de Ciências da Vida da UNEB, Campus I. Rua Silveira Martins, 2555 - Cabula. Salvador (BA), Brasil. CEP 41150-000. E-mail: luanabacelar.farma@gmail.com
} 


\section{INTRODUÇÃO}

A enterocolite neutropênica (EN) ou tiflite é uma complicaçáo grave da neutropenia caracterizada por ulceração e inflamação segmentar com necrose de íleo, ceco e cólon ascendente; sua patogênese é pouco conhecida e provavelmente multifatorial. Os principais elementos no início da doença parecem ser lesão da mucosa intestinal, juntamente com neutropenia. Essas condiçóes iniciais levam a edema intestinal e a uma superfície da mucosa rompida, que se torna mais vulnerável à translocação bacteriana ${ }^{1-3}$.

Com a utilização de regimes quimioterápicos intensivos, a EN tem se tornado uma complicação cada vez mais frequente na terapia oncológica, principalmente nos pacientes neutropênicos. Enteropatia necrotizante, tiflite ou síndrome ileocecal foram outros sinônimos inicialmente utilizados na literatura, na descrição de relatos de casos isolados. A imunossupressão induzida por neutropenia, combinada com toxicidade quimioterápica, infiltração tumoral, hemorragia intramural e reação inflamatória, leva à lesão da mucosa direta, até danos necrosantes e translocação microbiana ${ }^{2,3}$.

A verdadeira incidência de EN é desconhecida. Uma revisão sistemática publicada em 2005 sugeriu uma incidência de 5,3\% em adultos hospitalizados, para tratamento de neoplasias malignas sólidas ou hematológicas, e em pacientes com aplasia de medula. A mortalidade é de aproximadamente $50 \%$ na média, podendo atingir $100 \%$, pois a maioria dos pacientes é imunossuprimida ${ }^{4}$. Afeta crianças e adultos com neutropenia grave, sendo uma condição clínica inicialmente descrita em pacientes pediátricos leucêmicos ${ }^{3,5}$.

O estado de imunossupressão refere-se a uma alteraçáo do sistema de defesa do hospedeiro, que leva ao aumento da suscetibilidade às infecçôes; a neutropenia predispóe o indivíduo a infecçôes graves. Os pacientes neutropênicos constituem uma população heterogênea, que necessita de parâmetros adicionais para ajudar a definir o risco real da infecção e personalizar uma abordagem mais específica a cada paciente nessa categoria. Os fatores de risco para infecção associada à neutropenia incluem idade avançada, performance clínica ou nutricional precárias, contagens hematológicas baixas em condições basais e mínimas depois do primeiro ciclo de quimioterapia em altas doses ${ }^{1}$.

$\mathrm{O}$ reconhecimento e o tratamento precoce são vitais para evitar resultados adversos. Os pacientes geralmente apresentam sintomas gastrointestinais em um contexto de neutropenia, comumente após quimioterapia, com espessamento da parede intestinal e amostras microbiológicas positivas ${ }^{2,6}$.
Embora a EN seja uma condição clínica ocasionada como evento adverso de medicamentos, estes sáo insumos indispensáveis na assistência à saúde, sendo a tecnologia sanitária mais utilizada no cuidado dos usuários. Os medicamentos são essenciais por preservarem a vida, melhorarem a saúde e a resolubilidade dos serviços de saúde, isso graças às suas finalidades profilática, curativa, paliativa ou de diagnóstico. Entretanto, seu uso oferece risco para a ocorrência de efeitos indesejados, por vezes inesperados, podendo causar danos que vão desde o prolongamento da hospitalizaçáo, necessidade de intervençōes diagnósticas e terapêuticas, ou até mesmo óbito. Por tais razôes, devem ser considerados os aspectos de segurança, eficácia e qualidade dos medicamentos ${ }^{7}$.

$\mathrm{O}$ interesse atual na EN resulta de um aumento da sua prevalência na população adulta, como complicação de esquemas agressivos de quimioterapia cada vez mais utilizados não só em neoplasias hematológicas, mas também em neoplasias sólidas. Mais recentemente, têm sido descritos alguns casos em associação com neoplasias sólidas, nomeadamente testículos, pulmão, cólon, mama etc. ${ }^{8}$. Trata-se de uma toxicidade importante que pode levar à piora do quadro clínico do paciente com câncer, levando-o à morte, mas que pode ser tratada se descoberta precocemente. Sendo assim, diante do exposto, o estudo visa a analisar um caso raro de $\mathrm{EN}$ em um paciente com tumor sólido, além de contribuir significativamente para discussóes que envolvem essa toxicidade, bem como a participação da equipe de saúde no desfecho clínico.

Refere-se a um estudo descritivo de caráter narrativo; ou seja, um relato de caso baseado em coleta de informaçóes de um paciente, via prontuário eletrônico, internado em um hospital filantrópico de Salvador - BA, no período de maio de 2018, com diagnóstico de câncer de mama, evoluindo com EN após tratamento com quimioterapia adjuvante.

O relato envolveu uma busca via prontuário eletrônico, restrito aos pesquisadores identificados na equipe, com a utilização dos dados, exclusivamente, para a execução do presente trabalho. A metodologia realizada no caso clínico foi submetida à apreciação do Comitê de Ética em Pesquisa e aprovada sob o número de parecer 4.013.581.

\section{RELATO DO CASO}

Paciente do sexo masculino, 75 anos, $86 \mathrm{Kg}, 1,62 \mathrm{~m}$, hipertenso, diabético, dislipidêmico, com insuficiência renal crônica e sedentário. Em 2018, notou presença de nódulo em mama esquerda. O estudo histológico revelou carcinoma ductal invasivo de grau histológico pouco diferenciado (grau 3) e, com relação à biologia do tumor, o resultado do exame demonstrou positividade para os 
receptores de estrogênio e progesterona, expressão de human epidermal growth factor receptor-type 2 (HER-2) positivo e antígeno de proliferação celular Ki-67 de 30\%.

Dessa forma, foi diagnosticado com câncer de mama, indicação médica de tratamento adjuvante com mastectomia simples e quimioterapia com docetaxel $(75$ $\mathrm{mg} / \mathrm{m}^{2}$ ), carboplatina (AUC 6) e trastuzumabe $(8 \mathrm{mg} /$ $\mathrm{Kg}$ - dose de ataque e $6 \mathrm{mg} / \mathrm{Kg}$ - dose de manutenção) repetidos a cada 21 dias, em um total de seis ciclos. Negava história familiar de câncer de mama e realização de qualquer tipo de tratamento quimioterápico.

Ao iniciar o primeiro dia de quimioterapia, em nível ambulatorial, retornou após cinco dias com queixa de dor em "cisto no pescoço" de longa data, previamente indolor, além de relatar aumento de volume da lesão cervical. Ao exame físico, observaram-se melena e hipocalemia; no segundo dia de internaçáo, obteve melhora da dor na regiáo occipital, porém com presença de hematoquezia; evoluiu em vigilância infecciosa e renal. No terceiro dia de internaçáo, o paciente evoluiu com quadro de hipotensấo, dor e distensão abdominal; o leucograma mostrou leucopenia absoluta com $530 / \mathrm{mm}^{3}$ e neutropenia absoluta, grau $4\left(170 / \mathrm{mm}^{3}\right)$.

O resultado da hemocultura evidenciou positividade para Staphylococus epidermidis, sepse e diagnóstico de EN. A conduta médica consistiu na introdução de antibioticoterapia sistêmica e fator estimulador de colônias de granulócitos humanos. No quarto dia de internação, o paciente foi a óbito associado ao quadro de EN ou tiflite.

\section{DISCUSSÃO}

O câncer de mama é o tipo de câncer mais comum entre as mulheres no mundo e no Brasil; depois do câncer de pele não melanoma, responde, atualmente, por cerca de $28 \%$ dos casos novos de câncer em mulheres. O câncer de mama também acomete homens, porém, é raro, representando menos de $1 \%$ do total de casos da doença. Relativamente raro antes dos 35 anos, acima dessa idade, sua incidência cresce progressivamente, especialmente após os 50 anos. Estatísticas indicam aumento da sua incidência tanto nos países desenvolvidos quanto nos em desenvolvimento?.

O tratamento do câncer de mama é complexo e depende do estadiamento clínico tumoral, das características da neoplasia e das condições clínicas do paciente. A quimioterapia é uma alternativa de tratamento indicado frequentemente, que inclui uma grande gama de medicamentos antineoplásicos e pode ser iniciada nos contextos neoadjuvante, adjuvante ou metastático. A quimioterapia neoadjuvante é conhecida como primária ou pré-operatória, sendo utilizada por pacientes com câncer de mama localmente avançado e operável, tendo como principal objetivo reduzir o tumor primário e permitir a conservação da mama. A quimioterapia adjuvante é utilizada após o procedimento cirúrgico com intuito curativo e, juntamente com a terapia hormonal em casos selecionados, prolonga a sobrevida do paciente; os principais quimioterápicos utilizados no tratamento do câncer de mama são os antracíclicos e os taxanos ${ }^{10,11}$.

O protocolo quimioterápico proposto para o paciente, composto por carboplatina, docetaxel e trastuzumabe, foi citado em um relato de caso, no qual uma mulher com tumor de mama HER-2 tratada com esse esquema evoluiu com neutropenia grave ${ }^{12}$. Em um outro estudo clínico realizado entre 50 mulheres japonesas com diagnóstico de câncer de mama em tratamento com esse mesmo protocolo, 18,36\% apresentaram toxicidade hematológica e 34\% tiveram redução da dose ou descontinuação do tratamento em virtude das reaçóes adversas ${ }^{13}$. Sendo assim, os esquemas quimioterápicos mais frequentemente associados à neutropenia foram combinaçôes de platina e taxano (carboplatina e docetaxel; carboplatina, docetaxel e trastuzumabe; carboplatina e nab-paclitaxel), o que fomenta a relaçáo do tratamento quimioterápico do paciente em questáo com a toxicidade apresentada $a^{5,11}$.

Apesar da evolução contínua na prevenção de infecção, a neutropenia febril mantém-se como uma complicação frequente em pacientes oncológicos e é fator limitante na administração da terapêutica sistêmica antineoplásica. Sendo as mucosas do sistema gastrintestinal constituídas por epitélio em constante proliferação, são também as mais afetadas por terapêutica citotóxica antineoplásica ${ }^{14}$. É incomum encontrar a EN em pacientes com câncer de mama. O período de neutropenia relacionada à quimioterapia é geralmente de curta duração nesses pacientes. No entanto, nos últimos anos, a condição tem sido relatada em pacientes submetidos à quimioterapia baseada em taxano. É sabido que esses agentes têm um grande potencial para causar extensas alteraçóes inflamatórias na mucosa ${ }^{5}$.

Após o internamento, o paciente evoluiu com quadro de dor e distensão abdominal, além de hematoquezia e melena. Alguns autores ${ }^{6,3}$ relataram que as características clínicas de EN incluem febre e dor abdominal, porém distensão abdominal, inchaço e diarreia também foram relatados como sinais e sintomas associados. Melena ou hematoquezia são geralmente formas menos comuns de apresentação, o que torna difícil definir um diagnóstico precoce com condutas terapêuticas bem definidas.

E, em virtude da sua apresentação inespecífica, a EN pode imitar muitos outros diagnósticos, porém a confirmação geralmente envolve achados de febre, dor abdominal, neutropenia e espessamento da parede 
abdominal. Embora essa condição ocorra principalmente em pacientes gravemente imunossuprimidos ou mielossuprimidos com leucemia, também foi observada em indivíduos com outras neoplasias avançadas que receberam quimioterapia imunossupressora ${ }^{5}$.

A evolução do quadro sugeriu EN, sendo iniciado tratamento com antimicrobiano de amplo espectro e fator de crescimento. O manejo da EN parece controverso e, em razáo de melhorias nos cuidados de suporte, estudos ${ }^{2,3,5}$ relataram o sucesso do tratamento náo cirúrgico. $\mathrm{O}$ consenso é a administração imediata de antibióticos que devem cobrir patógenos gram-positivos, gram-negativos e anaeróbicos. Outros trabalhos ${ }^{3,5,12}$ também sugerem a vantagem de usar fator estimulador de colônias de granulócitos em pacientes neutropênicos com sepse ou choque.

No caso do paciente em questáo, a presença de comorbidades e a idade foram os principais fatores complicadores do quadro de neutropenia, e o desfecho clínico resultou em óbito. O risco de complicaçóes graves depende principalmente da duração da neutropenia $(>7$ dias), da presença de comorbidades, como disfunção hepática ou renal, idade $>60$ anos; e, entre as principais situaçôes clínicas encontradas em pacientes neutropênicos gravemente enfermos, as infecçōes gastrointestinais foram as mais frequentes em $31 \%$, seguidas pelas respiratórias em $30 \%{ }^{15}$.

Com o advento de regimes quimioterápicos cada vez mais sofisticados, seu uso melhorou, significativamente, as taxas de sobrevivência ao câncer. Porém, os eventos adversos ao tratamento e os sintomas associados à doença tornam indispensável a preocupação com a qualidade de vida desses pacientes. Dessa forma, em se tratando de uma toxicidade rara e com alta taxa de mortalidade como a $\mathrm{EN}$, a equipe multiprofissional deve-se mostrar preparada para identificar os sinais e sintomas, e oferecer opçóes no tratamento para obter uma melhora na qualidade de vida $^{16}$.

\section{CONCLUSÃO}

A EN é uma condição clínica rara, de difícil diagnóstico e letal. Pacientes em tratamento quimioterápico estão mais propensos a quadros severos de leucopenia e, consequentemente, evoluem para EN. Sendo assim, deve ser enfatizada a necessidade de maiores discussóes acerca do tema, visto que o diagnóstico precoce é fundamental para otimizar medidas terapêutica.

Embora o desfecho clínico deste caso não tenha sido favorável, os achados do presente estudo permitiram contribuir de maneira significativa na tomada de decisão clínica, bem como melhorar indicadores associados à alta taxa de mortalidade da tiflite. Sendo assim, em virtude do notório aumento dos casos de EN, aponta-se como perspectiva a qualificação da equipe de saúde para a inserção de profissionais ainda mais especializados, capazes de contribuir e identificar os sinais e sintomas relacionados com toxicidades hematológicas, resultado de tratamentos quimioterápicos.

\section{CONTRIBUIÇÕES}

Luana Santana Bacelar, Martamaria de Souza Ferraz Ribeiro e Maria Teresita Bendicho contribuíram na concepçáo e/ou no planejamento do estudo; na obtençáo, análise e interpretação dos dados; assim como na redação e revisão crítica. Rosa Malena Fagundes Xavier, Elisama Andrade Miranda e Caroline Chagas Lopes contribuíram na redação e revisão crítica com contribuição intelectual. Todas as autoras aprovaram a versão final a ser publicada.

\section{DECLARAÇÃO DE CONFLITO DE INTERESSES}

\author{
Nada a declarar.
}

\section{FONTES DE FINANCIAMENTO}

Não há.

\section{REFERÊNCIAS}

1. Chabner BA, Longo DL, Lydie P, et al. Manual de oncologia de Harrison. 2. ed. Porto Alegre: Amgh; 2015. Capítulo 17, Carpenter SM, Vianello F, Poznansky MC. Neutropenia Febril; p. 200.

2. Saillard C, Zafrani L, Darmon M, et al. The prognostic impact of abdominal surgery in cancer patients with neutropenic enterocolitis: a systematic review and meta-analysis, on behalf the Groupe de Recherche en Réanimation Respiratoire du patient d'OncoHématologie (GRRR-OH). Ann Intensive Care. 2018;8(1):47. doi: https://doi.org/10.1186/s13613018-0394-6

3. Rodrigues FG, Dasilva G, Wexner SD. Neutropenic enterocolitis. World J Gastroenterol. 2017;23(1):42-7. doi: https://doi.org/10.3748/wjg.v23.i1.42

4. Gorschlüter M, Mey U, Strehl J, et al. Neutropenic enterocolitis in adults: systematic analysis of evidence quality. Eur J Haematol. 2005;75(1):1-13. doi: https:// doi.org/10.1111/j.1600-0609.2005.00442.x

5. Eulálio Filho WNM, Gonçalves Neto T, Vieira SC. Typhlitis in breast cancer patient using Docetaxelbased chemotherapy: case report. Rev Bras Mastologia. 2015;26(2):79-82. doi: https://doi.org/10.5327/ Z201600020010RBM 
6. Chow EJ, Bishop KD. Painless neutropenic enterocolitis in a patient undergoing chemotherapy. Curr Oncol. 2016;23(5):e514-e516. doi: https://doi.org/10.3747/ co.23.3119

7. Duarte ML, Batista LM, Albuquerque PMS. Notificaçóes de farmacovigilância em um hospital oncológico sentinela da Paraíba. Rev Bras Farm Hosp Serv Saúde. 2014;5(1):7-11.

8. Cruz A, Lima SC, Barroso S, et al. Enterocolite neutropénica em doente transplantado hepático. J Port Gastrenterol. 2008;15(1):16-9.

9. Instituto Nacional de Câncer Jose Alencar Gomes da Silva [Internet]. Rio de Janeiro: INCA; [data desconhecida]. Tipos de câncer: câncer de mama; [acesso 2020 abr 15]. Disponível em: https://www.inca.gov.br/tipos-de-cancer/ cancer-de-mama

10. Conte FM, Sgnaolin V, Sgnaolin V. Neutropenia associada ao tratamento do câncer de mama: revisão integrativa da literatura. Rev Bras Cancerol. 2019;65(3):e11307. doi: https://doi.org/10.32635/2176-9745. RBC.2019v65n3.307

11. Nascimento TG, Andrade M, Oliveira RA, et al. Neutropenia: ocorrência e manejo em mulheres com câncer de mama em quimioterapia. Rev Latino-Am Enfermagem. 2014;22(2):301-8. doi: http://doi. org/10.1590/0104-1169.3305.2416

12. Ghani EA, Kerr I, Dada R. Grade 3 trastuzumabinduced neutropenia in breast cancer patient. J Oncol Pharm Pract. 2014;20(2):154-7. doi: http://doi. org/10.1177/1078155213487394

13. Sugitani I, Ueda S, Sakurai T, et al. Neoadjuvant chemotherapy with trastuzumab, docetaxel, and carboplatin administered every 3 weeks for Japanese women with HER2-positive primary breast cancer: efficacy and safety. Int J Clin Oncol. 2017;22(5):880-6. doi: http://doi.org/10.1007/s10147-017-1136-8

14. Atalaia G, Vasconcelos P, Bragança N. Neutropenia febril. Rev Clin Hosp Prof Dr Fernando Fonseca. 2015;3(1):13-9.

15. García Cruz C, Vivar Maldonado J, Frías Toral E, et al. Mortalidad de pacientes oncológicos vinculados a neutropenia. Rev Oncol Ecu. 2019;29(1):12-26. doi: https://doi.org/10.33821/279

16. Andrade V, Sawada NO, Barichello E. Qualidade de vida de pacientes com câncer hematológico em tratamento quimioterápico. Rev Esc Enferm USP. 2013;47(2):355-61. doi: http://doi.org/10.1590/S008062342013000200012 